\title{
Safety and effectiveness of BCG vaccination in preterm babies
}

Sudhin Thayyil-Sudhan, Ashok Kumar, Meharban Singh, Vinod Kumar Paul, Ashok Kumar Deorari

\begin{abstract}
Aim-To assess the cell mediated immune response to BCG vaccine in preterm babies.

Methods-Sixty two consecutive preterm babies born at $<35$ weeks of gestation were randomly allocated into two groups. Babies in group A were vaccinated early at 34-35 weeks and group $B$ were vaccinated late at 38-40 weeks of postconceptional age. The two groups were similar in terms of: gestational age (mean (SD) 33.1 (1.1) and 33 (1.2) weeks, respectively); birthweight 1583 (204) and 1546 (218) g; neonatal problems; socioeconomic status; and postnatal weight gain. The cell mediated immune response to $B C G$ was assessed using the Mantoux test and the lymphocyte migration inhibition test (LMIT) 6-8 weeks after BCG vaccination. Induration of $>5 \mathrm{~mm}$ after the Mantoux test was taken as a positive response.

Results-There was no significant difference in the tuberculin conversion rates $(80 \%$ and $80.7 \%$, respectively), positive LMIT (86.6\% and $90.3 \%$, respectively), or BCG scar (90.0\% and $87.1 \%$, respectively) among the two groups.

Conclusions-Prematurity seems to be an unlikely cause for poor vaccine uptake. Preterm babies can be effectively vaccinated with BCG at 34-35 weeks of postconceptional age, the normal time of discharge in a developing country.

(Arch Dis Child Fetal Neonatal Ed 1999;81:F64-F66)
\end{abstract}

Keywords: BCG; tuberculin test; lymphocyte migration inhibition test

BCG vaccination is compulsory in 64 countries and is officially recommended in a further 118 . In 10 randomised controlled trials conducted throughout the world since 1930, the overall protection afforded by BCG vaccination has ranged from $0-80 \%$. BCG vaccination seems to limit the multiplication and dissemination of the tubercle bacillus, rather than preventing infection in someone already exposed to the disease. This explains the $80-100 \%$ protection afforded by neonatal BCG vaccination against tuberculous meningitis reported in several case-control trials. ${ }^{1-4}$ In the UK 75 per cent of cases of tuberculous meningitis occur in children under 5 years of age who are thus in an age group that could have been protected by neonatal BCG vaccination. ${ }^{1}$

However, there are no guidelines regarding BCG vaccination in preterm babies who constitute about $8-10 \%$ of all neonates and who may not be immunocompetent to respond to BCG antigen. Our previous study had shown similar seroconversion rates in preterm babies and term babies following oral polio vaccination. ${ }^{5}$ In most studies on the efficacy of neonatal BCG vaccination, the preterm infants were not studied separately. To the best of our knowledge, there have only been three small studies conducted on BCG vaccination of preterm babies and they have reached conflicting conclusions. ${ }^{6-8}$

This study aimed to determine whether BCG vaccination given early to preterm babies at 34-35 weeks is as effective as when given late at 38-40 weeks of postconceptional age.

\section{Methods}

Ninety one preterm, appropriate for gestational age (AGA) babies of $<35$ weeks, consecutively born at the All India Institute of Medical Sciences between December 1995 and March 1997, were included in the trial. Critically ill babies, defined as those requiring assisted ventilation or parenteral fluids above two thirds of their total requirement; babies who underwent exchange transfusion; twins; babies who received immunoglobulins, steroids, INH prophylaxis; and babies with congenital malformations, were excluded from the study. Small for date babies were excluded because our previous work had shown poor uptake of BCG vaccine. $^{9}$

Twenty nine babies were thus excluded and the remaining 62 were randomly allocated (block randomisation with permuted blocks) into two groups after obtaining parental consent. Babies in group A were vaccinated early at 34-35 weeks and group B were vaccinated late at $38-40$ weeks of postconceptional age.

The two groups were similar in terms of gestational age (mean (SD) 33.1(1.1) and 33.0 (1.2) weeks), birthweight (1583 (204) and 1546 (218) g), neonatal problems, socioeconomic status and postnatal weight gain.

Danish 1331 (BCG) manufactured at Guindy according to the regulations of the State Serum Institute in Copenhagen, was administered intradermally in a dose of $0.1 \mathrm{ml}$. Tuberculin skin tests were done 6-8 weeks after vaccination using $0.1 \mathrm{ml}$ of 1 TU PPD RT 23. An induration of more than $5 \mathrm{~mm}$ (read between 48-72 hours after the intradermal injection) was taken as a positive response. Both BCG and tuberculin skin tests were administered and read by the principal author. Mantoux reading was double checked by an experienced immunisation nurse. 
Table 1 Tuberculin conversion, lymphocyte migration test (LMIT), and scar formation (vaccine uptake) after BCG vaccination of preterm babies

\begin{tabular}{|c|c|c|c|c|c|}
\hline & \multicolumn{2}{|c|}{ Group $A(n=30)$} & \multicolumn{2}{|c|}{ Group B $(n=31)$} & \multirow[b]{2}{*}{$p$ Value } \\
\hline & $n(\%)$ & $95 \% C I$ & $n(\%)$ & $95 \% C I$ & \\
\hline Positive tuberculin skin test & $24(80.0)$ & $61.4,92.3$ & $25(80.7)$ & $62.5,92.6$ & $\star^{\star} 0.8$ \\
\hline Positive LMIT & $26(86.6)$ & $69.3,96.2$ & $28(90.3)$ & $74.3,98.0$ & $\$ 0.7$ \\
\hline BCG scar & $27(90.0)$ & $73.5,97.9$ & $27(87.1)$ & $70.2,96.4$ & $\$ 1.0$ \\
\hline
\end{tabular}

${ }^{\star} \chi^{2}$ test (0.07) with Yates' correction; $§$ Fisher's exact test ( 2 tailed).

Group A: preterm babies vaccinated early at 34-35 weeks of postconceptional age.

Group B: preterm babies vaccinated late at $38-40$ weeks of postconceptional age.

The lymphocyte migration inhibition test (LMIT) was done on all the babies, 6-8 weeks after vaccination. Several studies have shown good correlation between the in vivo response measured by LMIT and the in vitro response measured by tuberculin skin test, following BCG vaccination. LMIT is reported to be more sensitive than the tuberculin skin test in assessing the cell mediated immune response to $\mathrm{BCG}$ vaccine. ${ }^{9-11}$

Blood anticoagulated with heparin was mixed with $2 \%$ gelatin in phosphate buffered saline taken in a silicon tube. Cells were allowed to migrate in Perspex chambers for 16-18 hours in minimal essential medium (MEM) containing $10 \%$ fetal calf serum with or without the antigen (PPD). A migration index of less than 0.8 was taken as a positive response, indicating a cell mediated immune response to BCG..$^{9-11}$

\section{Results}

One baby in group A died due to intraventricular haemorrhage and sepsis; the rest completed the study. There was no significant difference in the incidence of tuberculin conversion, positive LMIT, or BCG scar among the two groups $(\mathrm{p}>0.05)$.

Ninety per cent of the preterm babies vaccinated early and $87.1 \%$ of those vaccinated late had a scar or ulcer at the vaccine site (table 1 ), indicating successful vaccination. Eighty per cent of the babies in both groups had positive Mantoux conversion. Positive LMIT was seen in $86.6 \%$ of the early vaccinees and $90.2 \%$ of the late vaccinees, indicating a cell mediated immune response to BCG vaccination. Mean induration after the tuberculin skin test was also

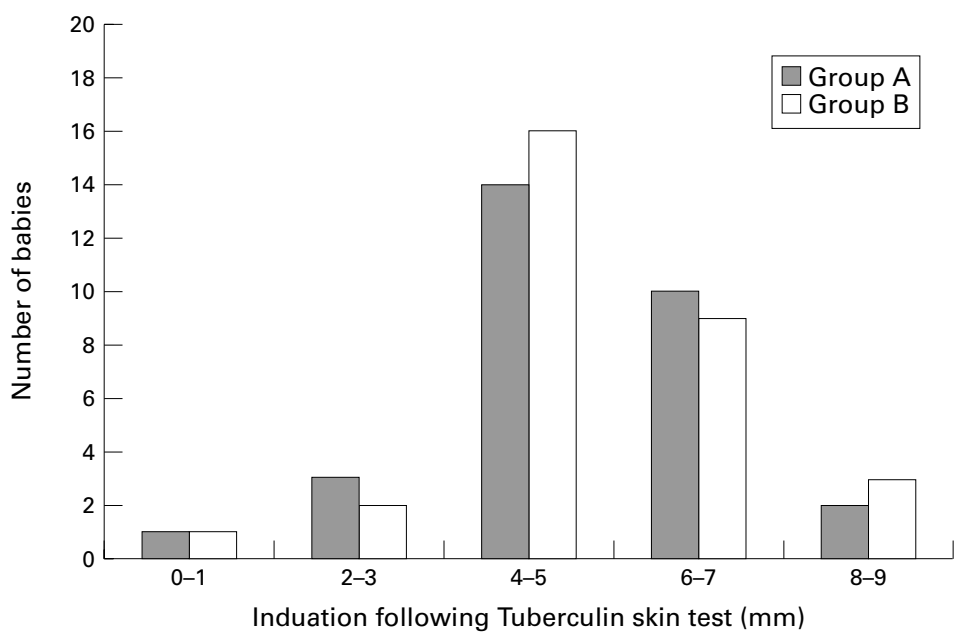

Figure 1 Induration diameter of tuberculin skin test in preterm babies studied. similar, $5.27 \mathrm{~mm}$ (SD1.8) in group A and 5.26 $\mathrm{mm}$ (SD1.6) in group B. None of the babies had induration of more than $10 \mathrm{~mm}$ (fig 1).

All the babies with scars except one $(98.1 \%)$ were positive for LMIT. Of the seven scar negative babies, three $(42.8 \%)$ were positive for LMIT. The tuberculin test and LMIT showed good correlation $(\mathrm{r}=0.84)$. The tuberculin skin test showed a high degree of sensitivity and specificity when the cutoff for a positive Mantoux reaction was taken as $5 \mathrm{~mm}$, considering LMIT as the gold standard for immune response. None of the babies had any complications following the vaccination. Small axillary lymph nodes $(<5 \mathrm{~mm})$ were found in $36 \%$.

\section{Discussion}

The study shows similar uptake and cell mediated immune responses against BCG vaccine in preterm babies vaccinated early at 34 weeks and late at 38-40 weeks of postconceptional age. Delaying the age of vaccination did not seem to improve the conversion rates and might increase the number of missed vaccination opportunities. The conversion rates in preterm babies are similar to those reported for term neonates. ${ }^{12}{ }^{13}$ None of the babies had lymph nodes of more than $5 \mathrm{~mm}$ or suppuration.

Dawadu et al reported a tuberculin conversion rate (induration $>5 \mathrm{~mm}$ ) of $83 \%$ in 12 preterm babies vaccinated at birth compared with $88 \%$ conversion rates in eight preterm babies vaccinated at 38-40 weeks of postconceptional age. ${ }^{6}$ The differences, however, were not significant and the findings show that BCG vaccine can be given effectively at 34-35 weeks of postconceptional age in preterm babies. Small for date babies, those who had respiratory distress syndrome, sepsis, jaundice, apnoea, and those who had congenital malformations, were excluded from this study.

In a recent study Sedaghatian and Kardouni examined the tuberculin response and scar formation following BCG vaccination of preterm babies at birth. ${ }^{7}$ The study included 289 preterm babies, but only $70(24.2 \%)$ completed the study. The only exclusion criteria was major congenital malformation. Thirty two per cent of the babies had no BCG scar and only $20 \%$ had tuberculin conversions of more than $5 \mathrm{~mm}$. The authors therefore concluded that routine BCG vaccination in preterm babies was not indicated. Poor follow up and inclusion of sick and small for date babies might have influenced the results of their study. No in vivo tests were used to elicit the cell mediated immune response in this study.

In their second study Sedaghatian et al compared the tuberculin skin tests and scars following BCG vaccination in 36 preterm babies vaccinated at birth (group 1), 16 preterm babies vaccinated at 40 weeks (group 2 ), and 20 full term babies vaccinated at birth (group 3). The only exclusion criterion used in this study was a multiple congenital anomaly. Thirty three per cent, $25 \%$, and $25 \%$, respectively, of babies in groups 1,2 , and 3 produced negative Mantoux reactions. BCG scars were absent in $55 \%, 44 \%$, and $40 \%$, respectively. 
The differences in the Mantoux conversion rates and BCG scar between the three groups were not significant $(p>0.4)$. The authors subsequently did a multivariate analysis and found that birthweight was significantly associated with tuberculin skin test reaction. It was therefore concluded that routine BCG vaccination at birth of preterm babies of $<33$ weeks is not indicated, until further studies are done. ${ }^{8}$

A Mantoux conversion rate of $81.2 \%$ was observed in both term neonates and low birthweight babies vaccinated at birth in a recent study by Ferreria et al. ${ }^{12}$ These conversion rates are very similar to those in our study.

Correlation between the protection afforded by BCG vaccination and tuberculin skin responses is controversial. However, skin test responses and in vitro assays of cell mediated immunity are probably the best surrogates of the efficacy of BCG vaccine, currently available. ${ }^{14}$

Prematurity itself seems to be an unlikely cause for poor vaccine uptake and cell mediated immune response. Thus we conclude that babies born at 34 weeks can be safely vaccinated with BCG within days after birth, which is the time when they are discharged from hospital in developing countries.

We thank Dr Richard Hancock, Queen's Hospital, Burton, UK for statistical assistance and Mr Gopal Singh for assisting with laboratory work.
1 Clarke A, Rudd P. Neonatal BCG immunisation. Arch Dis Child 1992;67:473-4.

2 Colditz GA, Brewer TF, Berkey CS, et al. Efficacy of BCG vaccine in the prevention of tuberculosis: metaanalysis of the published literature. $\mathcal{F A M A} 1994 ; 271: 698-702$.

3 Thilothammal N, Krishnamuthy PV, Runyan DK, Banu K. Does BCG vaccine prevent tuberculous meningitis. Arch Dis Child 1996;74:144-7.

4 Filho VW, de Castilho EA, Rodriques LC, Huttly SRA. Effectiveness of BCG vaccination against tuberculous meningitis: a case-control study in Soa Paulo, Brazil. Bull WHO 1990;68:69-74.

5 Thayyil-Sudhan S, Singh M, Broor S, Xess I, Paul VK, Deorari AK. Is zero dose oral polio vaccine effective in preterm babies? Ann Trop Paediatr 1998;18:321-4.

6 Dawadu AH. Tuberculin conversion following BCG vaccination in preterm infants. Acta Pediatr Scand 1985;74:5648 .

7 Sedaghatian MR, Kardouni K. Tuberculin response in preterm infants after BCG vaccination at birth. Arch Dis Child term infants after

8 Sedaghatian MR, Hashem F, Hossain MM. Bacille Calmette Guerin vaccination in preterm infants. Int $\mathcal{F}$ Tuberc Lung Dis 1998;2:679-82.

9 Singh M, Manerikar S, Malaviya AN, et al. Immune status and BCG vaccination in new-born infants with IUGR. Clin Exp Immunol 1976;26:173-5.

10 Kathipari K, Seth V, Sinclair S, Arora NK, Kukreja N. Cell mediated immune response after BCG vaccination as a
determinant of optimum age of vaccination. Indian $7 \mathrm{Med}$ Res 1982; 65:508-11.

11 Vijayalakshmi V, Devi PS, Murthy KJR, Rao DR, Jain SN. Cell mediated immune response in BCG vaccinated children Indian Pediatr 1993;30:899-903.

12 Ferreira AA, Bunn MM, Anna CCS, Ferreira MFC. BCG vaccination in low birth weight new-borns: analysis of vaccination in low birth weight new-borns: analysis of lymphocyte proliferation, IL-2 generation and intradern

13 Pinhata MMM, GoncalvesAL, Foss NT. BCG vaccination of Full-term infants with IUGR:Influence of immunisation age on the development of post vaccination delayed tuberculin sensitivity. Bull WHO 1993;31:41-6.

4 Comstock GW. Does the protective effect of neonatal BCG vaccination correlate with vaccine-induced tuberculin reactions Am f Respir Crit Care Med 1996;154:263-4 